\title{
Violence after discharge from forensic units in the safe pilot study: a prospective study with matched pair design
}

This article was published in the following Dove Press journal: Psychology Research and Behavior Management

\author{
Stål Bjørkly ${ }^{1,2}$ \\ Jon Magnus Wærstad' \\ Lars Erik Selmer' \\ Johnny Wærp' \\ Martin Bjørnstad' \\ John Vegard Leinslie' \\ Gunnar Eidhammer ' \\ Kevin S Douglas $1,3,4$ \\ 'Centre for Research and Education in \\ Forensic Psychiatry, Oslo University \\ Hospital-HF, Oslo N-0320, Norway; \\ ${ }^{2}$ Faculty of Health and Social Sciences, \\ Molde University College, Molde N-6402, \\ Norway; ${ }^{3}$ Department of Psychology, \\ Simon Fraser University, Burnaby V5A \\ IS6, BC, Canada; ${ }^{4}$ Centre for Research \\ and Education in Forensic Psychiatry, \\ Bergen Health Trust, Bergen 502I, \\ Norway
}

Correspondence: Stål Bjørkly

Centre for Research and Education in Forensic Psychiatry, Oslo University Hospital-HF, P.O Box 2110, Molde 6402, Norway

Tel +4747849800

Fax +4771214100

Email stal.bjorkly@himolde.no
Objective: This paper reports on a prospective naturalistic study of violent recidivism after discharge from forensic mental health. Main aims were to find predictors of violence and to test the feasibility of a matched pair design for this purpose.

Methods: Patients from the Safe pilot project $(n=18)$ and a group of controls $(n=18)$ were matched on 10 variables, such as diagnosis, seriousness of violence, setting after discharge, and risk management plans. All the Safe pilot patients had been through repeated measurement of dynamic risk factors of violence the year before discharge to develop efficient risk management plans for use after discharge. We wanted to test whether violent recidivism during follow-up would be lower and less serious in the Safe pilot group.

Results: We found no significant between-group difference concerning number of patients with violent recidivism. However, the Safe pilot patients had significantly lower rates of violence and fewer severe violent episodes. In the control group, there was a significant association between a high number of risk management plans and high rates of violence. There was a statistical trend for the opposite association in the Safe pilot group.

Conclusion: We discuss this in terms of a possible gap between the development and implementation of plans.

Keywords: forensic mental health, discharge, violence, matched pair

\section{Introduction}

A main goal in secure forensic mental health services is to reduce violence within facilities and violent recidivism by patients after discharge. Hence, it is an urgent task to develop and validate assessment and prevention processes with respect to violent recidivism after forensic care.

There have been many studies of forensic patients discharged to the community, typically under some form of conditional supervision. Fazel and colleagues ${ }^{1}$ systematic review showed high rates of reoffending after patients' discharges from secure hospitals in England and Wales, and in Sweden and Norway. Studies from the UK have found that around $7 \%$ of secure hospital patients had committed grave violent offenses that potentially qualified for life sentences within two years of release. ${ }^{2,3}$

Most studies show violent recidivism rates of patients on conditional discharge under $20 \%{ }^{4}$ However, there is a substantial range. In the review by Hayes et al, ${ }^{4}$ primarily of US, Canadian, and UK studies, violent reconviction rates during conditional discharge were 6-15\%, depending on follow-up lengths and other study 
differences. For a broader variable - violent re-offending the range was $4-20 \%$. Similar rates of violent recidivism have been observed in non-UK European studies. ${ }^{5,6}$

There are numerous studies of conditionally discharged forensic patients with very low violent recidivism rates (ie, under $2 \%$ ), ostensibly showing support for the efficacy of conditional discharge in stemming violence. ${ }^{7-9}$ Although this might indeed be true, such optimism must be tempered by several factors. First, how violence is measured can drastically impact violent recidivism rates. To illustrate, consider research reported by Doyle and colleagues. ${ }^{10}$ They followed all 387 medium secure forensic patients discharged over the course of one year in England and Wales. They measured violence at six months and one year, post-discharge. Using official recidivism outcome data (police registry), the violent recidivism rates at six months and one year were $1.6 \%$ and $2.8 \%$. When supplemented with data from care provider interviews, however, the rates were $14 \%$ and $22.5 \%$ over these same time frames.

Second, detected violence recidivism rates can vary not only as a function of the sources of information used to measure violence, but by a host of other methodological factors. For instance, Penney, Marshall, and Simpson ${ }^{11}$ suggested that the wide range of observed violent recidivism rates stems from the different types of measurement, sample types, and length of follow-ups used across studies. They emphasized that establishing reliable prevalence rates for violence after discharge from forensic hospitals should be prioritized in future research.

Even though Fazel and collaborators (2016) found that rates of reoffending for released hospitalized patients were lower than those for released prisoners, improvement in violent recidivism in patients discharged from forensic psychiatry remains urgent. The Norwegian findings from Fazel et $\mathrm{aL}^{1}$ concerning rates of readmission and reconviction were similar to research findings from other countries, as was the finding that substance abuse was the strongest predictor for all adverse events. ${ }^{12}$ However, this study used face-to-face interviews - a key methodological difference from registry-based studies - and results showed that this approach enhanced access to important clinical and psychosocial information. Compared to official records, patients' self-reports were more reliable for violent crime and significantly more accurate for drug-related crimes, but not for petty crimes. This indicates that intensive designs may be an important supplement to registry studies in order to obtain an in-depth understanding of the processes leading to violent recidivism.
A third factor that might limit optimism associated with low recidivism rates is that, without any kind of comparison group, it is simply not possible, from a methodological design perspective, to attribute causation to any interventions or supervision that transpires during postdischarge conditional release. Some research has been able to compare patients on conditional release who then were transferred to unconditional release. ${ }^{6}$ Violent reoffending increased after the transfer (13\% of patients vs 6.5 of patients), although this increase in violent re-offending was not statistically significant.

The present paper reports findings from a pre-study integrated into the Safe pilot project ${ }^{13}$ that is novel in terms of addressing some of these methodological factors. It was an intensive, repeated measures prospective design that also included a matched comparison group. To our knowledge, there are no such other matched pairs prospective follow up studies of forensic patients supervised in the community.

The pilot project was conducted in Norway with an intensive prospective repeated measurement design within four areas: Psychopathology and general level of functioning; risk assessment; risk management strategies; and recidivism. One main purpose of the risk assessment part was to develop and validate approaches and instruments for risk assessment and management of patients discharged from high and medium security psychiatric facilities. This was partially accomplished by tailoring and comparing dynamic risk items made especially for assessment of violence with items from tools that originally were made to measure insight, psychotic symptoms, dissociation and hopelessness in general psychiatry. The hypothesis was that items developed especially for assessments of violence risk would inform clinicians more accurately than those taken from instruments originally designed for assessment of a wider range of psychiatric symptoms and conditions.

The most relevant issues for the current pre-study, however, were to (1) do research on patients discharged from forensic psychiatry to general psychiatry and communitybased psychiatric services in Norway and (2) conduct a prospective study of violent recidivism after discharge from medium and maximum secure units in Norway. The research designs used so far have predominantly been register studies within longitudinal designs. Very few have used repeated measurement designs, and, to our knowledge, matched pair design has not been conducted before. This was confirmed in an advanced search of the literature in 
Ovid MEDLINE(R) and OVID Psyclit from 1946 to August 09, 2018, on prospective matched-pair design in follow-up studies after discharge from forensic units. Search terms were: Matched AND design OR control OR compar* AND Prevent* OR risk management OR risk reduction OR deter* AND Violen* OR homicid* OR assault* OR rape OR robber* AND Recidiv* OR reoffend* OR repeat offend* AND Forensic* OR security AND ward OR unit. The search string had 214 combinations of search terms. Three papers were found, but further analysis of them showed that they were not relevant because they did not have a prospective follow-up design that measured violent recidivism after discharge from forensic psychiatry. The complete search string and search history can be obtained by contacting the corresponding author.

Our motivation for choosing a pre-study with matched pair design was the following: Matching participants as close as possible on measurable characteristics might help control for random individual differences affecting the results, in particular observed violent recidivism. In our study, we aimed to compare actual violence during followup with the only intended difference between the matched pairs being whether they belonged to the Safe pilot group or not.

We were also aware of disadvantages in the matched pairs design: First, matching for similar and relevant characteristics is time consuming and particularly complicated in naturalistic prospective studies. This may be a challenge for internal validity. Second, the sample may be small because it is not easy to find a large sample that fits well with the characteristics being searched for, and this may threaten external validity. Third, the matching may result in leveling out differences that otherwise would have been important case characteristics and explanatory variables in, for instance, a case-control study. Despite these issues, we wanted to do a matched pair pre-study in forensic psychiatry in two out of four Norwegian regional healthcare trusts. One year before the matched control study was finished, there were 30 beds in high security and 135 beds in medium security in the two regional healthcare trusts that served a population of 4 million inhabitants. ${ }^{14}$

One important difference between the Safe pilot group and the control group was that a battery of dynamic risk factors was conducted at least three times during the year before discharge and one time after discharge for the Safe pilot patients. We wondered whether repeat measurement of dynamic risk factors could inform the development and maintenance of individualized risk management strategies.
Furthermore, we wanted to compare rates and severity of violent acts between the two groups. We hypothesized that violent recidivism during follow-up would be lower in the Safe pilot group, provided that the specific knowledge accumulated for Safe pilot patients was carried forward to the next treatment unit. At the same time, we were mindful of the complexity of investigating whether this type of clinical competency could be successfully transferred and then used by staff and patients in the receiving facilities. Hence, we lowered the ambition of our main research interest to investigate whether we could find any difference between the intervention group and the control group during follow-up. This fits well with the pre-study design. Thus, the main aims of the present research were the following:

1. Report findings from the comparison of violent recidivism after discharge from forensic psychiatry between Safe pilot patients and matched control patients;

2. Test the feasibility and potential benefit of the matched control design for this type of prospective follow-up research.

\section{Materials and methods}

This naturalistic prospective study involved one maximum security ( $n=10$ beds) and four medium secure wards $(n=48$ beds) in the southern and western parts of Norway. The investigation was conducted with a matched pair design and lasted for 6 years. The Safe pilot project was approved concerning written informed consent by the Regional Committee for Medical and Health Research Ethics South East in Norway. The study was conducted in accordance with the Declaration of Helsinki.

\section{Participants}

Patients in the control group $(n=18)$ were matched with the Safe pilot group on the following variables: diagnosis, age, gender, ethnicity, most severe previous violence, number of admissions to psychiatric wards before the actual discharge, and type of treatment context after discharge. For two of the matched variables, age at transfer and follow-up time after discharge, we failed to recruit controls with exactly identical values to match a Safe pilot patient. The distributions of these inclusion factors were tested to rule out presence of significant differences between the intervention and the control group. The mean age at discharge was $32.39(S D=7.75)$ for the Safe pilot group and 33.56 
( $S D=7.96)$ for the control group. Results from a paired samples $t$-test showed no significant difference for age between the groups, $t(34)=-1.43, p<0.17, n s, 95 \% \mathrm{CI}$ $(-2.88--0.55)$, nor for follow-up time after discharge (Safe pilot group: $M=23.56$ months, $S D=16.07$ and for the control group: $M=28.67, S D=12.27), t(34)=-0.1 .17$, $p<0.257, n s, 95 \%$ CI $(-14.30-4.08)$. To make sure that there was no difference within pairs, follow-up time after discharge was adjusted to be similar in months within each pair of patients by using the follow-up time for the patient with the shortest period after discharge.

Each sample consisted of 3 women and 15 men. Their ethnicity was Norwegian (24 patients), Northern European except Norwegian (2), African (8), and Asian (2). Twentyfour had Norwegian citizenship and 12 had foreign citizenship. According to the ICD-10 criteria $^{15}$ (WHO, 1992), $83 \%$ of the samples had a main diagnosis within the schizophrenia spectrum disorders (22 paranoid schizophrenia, 4 hebephrenic schizophrenia, and 4 schizoaffective disorder patients). The other diagnoses were emotionally unstable personality disorder ( 2 patients), post-traumatic stress disorder (2) and Asperger's syndrome (2). Twentyeight patients had one or more comorbid drug dependence diagnoses.

The distribution of how many times the patients had been admitted to forensic inpatient treatment before the actual discharge was: No previous admittance, 6 patients; 1 to 3 times, 14 patients; 4 to 6 times, 2 patients; and over 6 times, 14 patients. The most serious violent episode before the current discharge was severe physical assault (30 patients) and homicide or homicide attempt (6 patients). Fifteen patients were discharged to community housing with partial staff follow-up; 12 patients were transferred to community living with staff presence $24 / 7$; and 8 were discharged to locked wards in general psychiatry. One patient in the Safe pilot group moved back to his apartment without any regular follow-up, apart from ordinary community health and social services on request from the patient. His matched control was discharged to community housing with partial staff follow-up, which is not very different from the living context of the Safe pilot patient.

\section{Research design}

This pairwise matched control design tested the following independent or predictor variables: Safe pilot group versus control group, violent versus non-violent group, age at discharge, follow-up time, chlorpromazine dosage per day, and number of risk management plans. The dependent variable was violent episodes operationalized as verbal threats, physical threats, physical assault, homicide attempt, homicide, and total of all violent episodes.

\section{Measures}

Violence was defined as intentional attempts at, threats of, or actual and intended infliction of bodily injury or harm on another person. The main criteria in this definition are from the MacArthur Violence Risk Assessment Study: ${ }^{16}$ physical assaults leading to physical injury in another person; the use of a dangerous object/weapon against another person; threats of using a weapon against another person; and the use of physical force in connection with sexual offenses. Physical and verbal threats that clearly implied an imminent physical assault were included in our study. Damage to property and harm to self was not accepted as violence, and fire-setting was only included if it was done with the intent to cause bodily harm to other people.

The following inclusion criteria for matched pairs were retrieved from ward reports and medical records in the electronic journal system: diagnosis, age, gender, ethnicity, most severe violence before discharge, and number of stays in a psychiatric ward. Type of treatment unit and follow-up time after discharge were recorded by the project researchers. Antipsychotic medication dosage at discharge was converted for each patient to chlorpromazine equivalents per day. Information about type and rate of risk management strategy plans for each patient was found by scrutinizing ward reports and medical records in the electronic journal system and confirmed by interviews with clinicians in charge of the patients during follow-up. The same sources were used to retrieve violent recidivism data. Observed violence during follow-up included verbal threats, physical threats, physical assault, and homicide attempts.

There were four types of risk management plans. Criterion-triggered intervention (CtI) originated in the Safe pilot project. ${ }^{13}$ It is composed of assessment of warning signs of violence and situations or interactions that would increase risk of violence. This information was the basis of making one or more predefined intervention plans to mitigate the risk by early intervention. The Early Recognition Method (ERM) is a similar approach, but it also has a structure involving weekly feedback and discussion with a patient concerning use of warning signs as triggers for coping behaviours or failure. ${ }^{17}$ The type of crisis plan used in Norway has a stronger emphasis on 
what to do when a crisis emerges than on how to identify situations where these interventions should be used. The HCR-20-V3 approach is by far the most advanced of these risk management strategies. ${ }^{18}$ Both the use of risk formulation and risk scenarios in version 3 may be used as basis for developing individual risk management plans. Implementation of risk management strategies is organized around four preventive measures: Monitoring, Treatment, Supervision, and Victim safety planning. Patients with this type of intervention plan had been recruited after March 2014 because the Norwegian translation of HCR-20-V3 was released then. Some patients had more than one management plan, and the number of intervention types per patient was tested as an independent variable. The data was quality controlled by three of the authors and coded into SPSS files (IBM SPSS Statistics 25).

\section{Procedure}

In Norway there are two levels of forensic psychiatry services, medium and high security wards. The difference is mainly that high security wards have higher staff-patient ratios. The staff-patient ratio is 5:1 in high security and 31 in medium security wards. Recruitment of Safe pilot patients $(\mathrm{n}=18)$ and case control patients $(\mathrm{n}=18)$ was based on discharge decisions by the clinicians in the secure wards. The matching was conducted at the individual level so that each Safe pilot study patient had a matched control patient.

\section{Statistical analysis}

Normal distribution of data was controlled for with interpretation of P-P plots. Paired samples $t$-test was used for comparison of matched data with normal distribution. McNemar's test for binomial distribution was applied for dichotomous data for matched pairs. For non-normal score distributions, we used independent samples Moses Test of Extreme Reactions for the difference between two independent groups in the extremity of scores and Mann-Whitney $U$ test. Chi-square test $\left(\chi^{2}\right)$ was applied for dichotomous data for independent groups. Multiple linear regression was used for multivariate analyses. Two stepwise four-factor models were tested for exploring possible independent variables associated with rates of violent episodes during follow-up. They were used to compare the Safe pilot group $(n=18)$ with the control group $(n=18)$, and the violent patients $(n=9)$ with nonviolent patients $(n=27)$. Estimates of Variation Inflation Factor (VIF) were used to test for multicollinearity between independent variables and interpretation of normal P-P plots for normality of residuals.

\section{Results}

Seven men and two women were violent during followup. They had the following characteristics: Norwegian origin, schizophrenia spectrum disorder, and comorbid drug dependence. Eight of these nine patients lived in community dwellings that provided either health personnel around the clock $(n=4)$ or only partial follow-up $(n=4)$. The remaining patient had been transferred to a locked general psychiatry ward. Concerning physical violence, a total of nine episodes were committed by four (11\%) patients. Only two of these patients were readmitted to secure forensic units - one Safe pilot patient (1 verbal threat) and one control-group patient (17 verbal and 17 physical threats).

\section{Safe pilot group compared with the control group}

The violence was skewed between the two groups, with one patient from the Safe pilot group having one episode of physical assault and three control group patients committing eight episodes of physical assault (Table 1).

There were two homicide attempts and both were committed by male patients from the control group. Only one of them was transferred back to the forensic unit. We found no significant difference concerning the number of violent patients in the Safe pilot group $(n=5)$ versus the matched control group $(\mathrm{n}=4)$ (McNemar's test, binomial distribution, exact significance, two-sided, $p=1.000$ ). However, controls had significantly more episodes and more severe violence ( $\mathrm{n}=55$ vs $\mathrm{n}=6$ episodes; $S=19,00$, $p=00.01$, Moses test of extreme reactions). This was the same for verbal threats ( $\mathrm{n}=23$ vs $4 ; S=18,00, p=00.01$, Moses test of extreme reactions), physical threats ( $n=24$ vs

Table I Rates of violence for the safe pilot group $(n=18)$ and the control group $(n=18)$

\begin{tabular}{|l|l|l|l|}
\hline & $\begin{array}{l}\text { Safe pilot } \\
\text { group }\end{array}$ & $\begin{array}{l}\text { Control } \\
\text { group }\end{array}$ & $\begin{array}{l}\text { Control } \\
\text { group }\end{array}$ \\
\hline Number of patients & 5 & 4 & 9 \\
Verbal threat & 4 & 23 & 27 \\
Physical threat & 1 & 24 & 25 \\
Physical assault & 1 & 6 & 7 \\
Homicide attempt & 0 & 2 & 2 \\
Total violence & 6 & 55 & 61 \\
\hline
\end{tabular}


1; $S=18,00, p=0$ 0.01, Moses test of extreme reactions), physical assaults ( $\mathrm{n}=6$ vs $1 ; S=19,00, p=00.01$, Moses test of extreme reactions), and homicide attempts (n $=2$ vs $0 ; S$ $=1,00, p=0$ 0.01, Moses test of extreme reactions).

One female and one male matched pair had violent episodes after discharge. The Safe pilot patients $(n=2)$ had only one verbal threat each, while one man in the matched control group had two physical threats, one physical assault, and one homicide attempt. The female matched control had two verbal threats, one physical threat, and one physical assault. Mean follow-up time after discharge for the violent patients was 23.56 months $(S D=16.07)$ for the Safe pilot group and 28.67 months $(S D=12.27)$ for controls. There was no significant difference between the groups for followup time after discharge, $t(34)=-1.173, p<0.257, n s, 95 \%$ CI (-14.304-4.082).

We found a trend towards a significant difference between the groups for chlorpromazine dosage per day at discharge, $M=792 \mathrm{mg}, S D=2303$ for the Safe pilot group and $M=949 \mathrm{mg}, S D=2278$ for the control group, $t(34)=$ $-1.173, p<0.090, n s, 95 \%$ CI $(-0.341-27.360)$. There was also a trend toward a significant difference for the number of risk management plans when comparing the Safe pilot group and the matched-control group. The Safe pilot group had a total of 17 plans compared to 12 plans in the control group, $t(34)=-1.917, p<0.072, n s, 95 \%$ CI $(0.045-$ 0.933). The only significant difference between the Safe pilot group and the control group concerning distribution of type of risk management plans was found for Criteriontriggered intervention: Safe pilot group $=6$ patients versus control group $=0$ (McNemar Test, binomial distribution, $p=0.031$ ) (Table 2).

We used multiple linear regression to explore possible explanatory variables and tentative models for violence recidivism within the limitations set by the matched control dataset. Two variables each were entered first in two separate analyses: follow-up time after discharge and number of risk management plans. They were stepwise combined with two other variables into the following models:

Model 1: Follow-up time after discharge, number of risk management plans, Chlorpromazine dosage at transition, and age at transfer.

Model 2: Follow-up time after discharge, number of risk management plans, Safe pilot group versus control group (dummy variable), and age at transfer.

The number of violent episodes was the dependent variable for testing both models. Model 1 yielded no significant associations. Using Model 2, the number of risk management plans entered together with the Safe pilot group versus the matched pair control group variable yielded the only significant model fit, $F=4.099, p=0.026$ adjusted $R$-square $=0.199(19.9 \%)$, and beta value $(\beta=.392, p=0.020,95 \% \mathrm{CI}=0.389-4.172)$. For patients in the Safe pilot group, there was a statistical trend towards a significant association between having a high number of risk management plans and low rates of violent episodes during follow-up $(\beta=-0.316, p=0.057,95 \% \mathrm{CI}=-7.582-$ 0.110 ). The standardized residuals for selected and unselected cases had an approximately normal distribution. No multicollinearity was found for number of risk management plans and Safe pilot group versus control group (VIF $=1.050$ for both variables). No other variable in the models was significant.

\section{Violent $(n=9)$ compared to non-violent $(n=27)$ patients}

The following variables were tested for possible differences between the violent and non-violent patients for violent episodes during follow-up: follow-up time after discharge, number of stays before transfer, chlorpromazine dosage at discharge, age at discharge, and the number of risk management plans at discharge. Only number of risk management

Table 2 Rates of patients and risk management plans for the Safe pilot group $(n=18)$ and the control group $(n=18)$

\begin{tabular}{|l|l|l|l|l|l|l|}
\hline \multicolumn{2}{c|}{} & \multicolumn{2}{l|}{ Safe pilot group } & \multicolumn{2}{l|}{ Control group } & \multicolumn{2}{l|}{ Control group } \\
\cline { 2 - 7 } & Patients & Plans & Patients & Plans & Patients & Plans \\
\hline No plan & 10 & 0 & 12 & 0 & 22 & 0 \\
One plan & 2 & 2 & 3 & 3 & 5 & 5 \\
Two plans & 3 & 6 & 3 & 6 & 6 & 3 \\
Three plans & 3 & 9 & 0 & 0 & 14 & 9 \\
Total patients with plans & 8 & & 6 & 9 & & \\
Total number of plans & & 17 & & & & 26 \\
\hline
\end{tabular}


plans $(M=1.89$ vs $M=0.33 ; S=25,000, p=0.001$, Moses test of extreme reactions) was significant. All patients with violence recidivism ( $\mathrm{n}=9,17$ plans) had at least one risk management plan compared to only five of the non-violent patients ( $\mathrm{n}=27,9$ plans) (Mann-Whitney $\mathrm{U}=222.000$, $p=0.001,2$-sided test). The difference was explained by the violent group having significantly more patients with ERM plans, 8 versus $3\left(\chi^{2}=19.244, p=0.001\right.$, Fischer's exact test, 2-sided test) and HCR-20 plans, 5 versus $2,\left(\chi^{2}=9.990\right.$, $p=0.006$, Fischer's exact test, 2-sided test).

We replicated the multiple linear regression procedure described previously for comparison between the violent group $(n=9)$ and the nonviolent group $(n=27)$. Testing of Model 1 and Model 2 resulted in no significant findings.

\section{Gender differences}

We found two significant gender differences. One for homicide attempt: no homicide attempt in females versus two attempts for males $(S=1.000, p=0.001$, Moses test of extreme reactions). The other one was for how many times the patients had been admitted to forensic inpatient treatment before the actual discharge: females $(n=6)$, all more than 6 stays; males $(n=30)$, first stay $(n=6), 1$ to 3 stays $(\mathrm{n}=14), 4$ to 6 stays $(\mathrm{n}=2)$, and over 6 stays $(\mathrm{n}=8)$, $\left(\chi^{2}=11.314, p=0.010\right.$, Fischer's exact test, 2-sided test $)$.

\section{Post hoc statistical power analysis}

Because of the small sample size and of our finding only one significant association in our multiple linear regression analysis, we conducted a post hoc statistical power analysis. The model included 4 variables which yielded a total $R$-squared of 0.199 . With the given sample size of 36 and alpha set at 0.05 , the power was $59 \%$ to detect a moderate effect size. It would take a sample size of 52 patients to obtain a statistical power of $80 \%$ to detect a moderate effect size.

\section{Discussion}

Our aims were (1) to report findings from a comparison of violence recidivism after discharge from forensic psychiatry between Safe pilot patients and matched control patients and (2) to test the feasibility and potential benefit of the matched control design for this type of prospective follow-up research. Our results must be interpreted within the limitations set by the small sample size and the impact of comprehensive matching of the Safe and control groups concerning explanatory variables for violent recidivism after transition.
To our knowledge, this is the first research with a matched paired design and monitoring of verbal and physical threats of violence during follow-up after discharge from forensic mental health facilities. Nine patients committed at least one type of violence. The finding of four (11\%) patients committing physical violence is similar to results from other studies of patients discharged from forensic facilities (e.g. ${ }^{11,19}$ ). Four (11\%) patients were readmitted to forensic mental health. Still, the stunning differences in the severity and frequency of violence that instigated the returns to forensic wards is not very easy to explain. One Safe pilot patient was readmitted due to a verbal threat, and another patient, for one verbal threat and one physical assault. In the control group, one patient had two physical threats, one physical assault, and one homicidal attempt. The other one had 17 verbal and 17 physical threats.

It appears that the threshold for re-admittance to forensic units was lower for Safe pilot patients than for controls, but we refrain from further speculation because a total of only four patients were sent back. Even if we keep the eight patients who were transitioned from forensic units to locked wards in general psychiatry out of the calculation, the return rate was $14 \%$, which is very low compared to results from other studies. For instance, Penney, Marshall, and Simpson ${ }^{20}$ found that $28 \%$ were readmitted within one year in a prospective investigation in Canada. Mean follow-up time for our samples was 26 months.

The low violence rate in our study might be explained by the fact that Norway invests considerable resources in community mental health services. Because the number of beds in general psychiatry has been reduced, follow-up after discharge from forensic facilities has been diverted to mental health community and outpatient treatment. This has resulted in an increased presence of well-qualified community mental health services and high staff-to-patient ratios in these facilities. The joint engagement of personnel in forensic units and community services throughout the transition period may also have made the transition period more seamless. For instance, in Norway it is common to use progression ladders whereby a structured, stepwise transition occurs starting with personnel from community services first meeting with a patient on a regular basis in the forensic unit to start building a relationship. ${ }^{13}$ Later, personnel from the forensic unit will follow the patient into community services to make a smooth transition. The progression ladder is a concrete 
description of the how, where, what, who, and when in this process. The main objective is to make this change easier for the patient and staff to handle. Still, this possible explanation must be interpreted with caution because we did not observe or monitor whether such structured transfer procedures were used in this research.

\section{Comparison of patients from the safe pilot group and the matched pair control group}

There was no significant difference between the two groups concerning number of patients with violent recidivism. However, the rates and severity of violent behaviour were significantly and substantially higher in the control group. The control group had over nine times more violent episodes than the Safe pilot group. One interpretation of this result is that the transfer of clinical knowledge about the Safe pilot patients' individual vulnerabilities and warning signs was more extensive and more accurately communicated during the transfer of these patients from the forensic wards. During the last year before discharge, the Safe pilot patients had been assessed with a battery of repeated measures of possible predictors of violence, such as delusions, hallucinations, insight, hopelessness, dissociation, resilience, and negative attitudes. The main purpose of these assessments was to build up a written profile of each individual's dynamic risk factors of violence, information that could then assist community health personnel in their efforts to integrate these patients into their new surroundings in the community. Although our researchers gathered evidence that the results of the dynamic risk factor assessments were communicated to the community-based personnel for each Safe pilot-patient, they did not then interview or conduct observational studies to learn how and to what extent this information was used after a patient was admitted to the new setting. Nonetheless, the comprehensive matching of the two groups concerning risk factors makes it a bit easier to suggest that the difference between the two groups concerning episodes of violence may have been associated with this transfer of clinical knowledge resulting from the repeated assessments.

After their violent episodes, five of the violent patients stayed in the community facilities where they were admitted after release from the forensic facilities, and four were readmitted to forensic facilities. Out of these four, only one of the Safe pilot group patients had committed a physical assault. The other Safe pilot patient who was readmitted to a medium secure unit had only made one verbal threat. It is easier to understand that the control group patient who had 17 verbal threats and 17 physical threats was transferred back to his previous forensic unit. Four patients in the control group had carried out physical assaults. Only two of them were readmitted to forensic services. Naturally, the low rate of readmission to locked forensic mental health facilities may be due to rejected applications of transfer to a forensic ward.

Still, it is worrying that only one of the two persons with a homicide attempt in the control group was readmitted to a forensic facility. Both of these two lived in community apartments when they committed their crimes. The one with only outpatient clinical follow-up once a week was readmitted to the forensic ward that he came from, while the other patient, who was supervised by personnel serving a cluster of apartments, still remained in the apartment after the violent episode. We assume, but do not know for sure, that suitable risk management strategies were implemented to avoid violent recidivism for this patient that remained with the community services. In sum, the finding of four patients $(11.1 \%)$ being readmitted to locked psychiatric wards is low compared to other studies. In contrast, for example, in the study of Penney and co-workers, ${ }^{11}$ the prevalence of violence was $23 \%$ one year after discharge.

Apart from rates and severity of violence, the only significant difference between the Safe pilot group and the control group was that six patients in the Safe pilot group had Criterion-triggered intervention plans whereas no patients in the control group had this type of risk management plan. This was because this specific intervention had been developed and implemented in the Safe pilot study only. Since the Criterion-triggered intervention is similar to the other risk management strategy plans found in both groups, we refrain from further interpretation of the role of the Criterion-triggered intervention in preventing violent recidivism. Still, we wanted to scrutinize whether the presence of risk management plans in general had any impact on violence. Our initial idea was that clinical use of such plans would mitigate risk of violence. However, when we tested this association with multiple linear regression analyses, we found a significant positive linear relationship between number of risk management plans and high rates of occurred violence during follow-up. However, a close to significant trend ( $p=0.057$ ) indicated that the rates of risk management plans were associated with reduced rates of violence in Safe pilot patients. We might hypothesize that the Safe pilot patients' 
risk management plans were better described and communicated in the transition process. Still, we do not know to what extent the plans were implemented and how the plans functioned in clinical practice. On the other hand, it is likely that patients with one or more risk management plans at discharge were assessed to have a greater need of this support than patients who were transferred without them. Thus, instead of being an indicator of a measure made to mitigate risk of violence, a high number of management plans may serve as a warning sign of pending violence, particularly if these plans are not implemented.

The finding of no significant difference between the Safe pilot group and the control group concerning chlorpromazine dosage at discharge does not mean that medication during follow-up was similar for the patients. Thus, it may only serve as a rough control variable in the analysis for level of medication treatment for psychotic patients, who constituted the large majority of the two groups. We simply do not know whether medication had any impact on reducing violence during follow-up. Still, we found a trend $(p=0.090)$ of a significant difference with a daily dosage of $949 \mathrm{mg}$ chlorpromazine in the control group versus $792 \mathrm{mg}$ in the Safe pilot group. As with the interpretation of risk management plans, this may be understood in two different ways. First, a higher dosage may indicate that despite the comprehensive matching that created very similar risk profiles between the two groups, patients in the control group may have had more severe psychotic symptoms. If so, then risk of violence may have been higher in the control group, and this may partly explain why there were more episodes of violence in that group. On the other hand, one can interpret this in favor of the Safe pilot group because even if the matched controls had a potentially more efficient violence prevention measure due to their medication, the Safe pilot-patients were only involved in about $10 \%$ of the violent episodes. Other research has found that pharmaceutical treatment has reduced the risk of being returned to a forensic hospital (e.g. ${ }^{21}$ ). In the bigger picture, diagnosis appears to differentially impact on readmission and reconviction. In a recent systematic review of patients discharged from medium secure forensic hospitals, Conlin and Braham ${ }^{22}$ found that persons with personality disorder had two times more reconvictions than persons with mental illness. Though pharmaceutical treatment may have a positive effect for psychotic patients, there is a substantial subgroup of forensic mental health patients that do not profit from it.

\section{Comparison of violent and non-violent patients}

The comparison between the 9 violent and 27 non-violent patients generated only one significant difference. The violent group had about 6 times more risk management plans at discharge from forensic wards than those who had no violent episodes during follow-up. As mentioned earlier, this main association was mostly explained by patients in the control group.

\section{The feasibility and potential benefit of the matched-control design for this type of prospective follow-up research}

Our literature search failed to find any publication reporting on tests of explanatory variables for violent recidivism after discharge from forensic mental health institutions with a matched pair design. We wanted to scrutinize whether the matching by a set of possible explanatory variables could reduce the number of covariates in the statistical analysis. The main aim was to develop a more accurate design for identifying between-group differences of violent recidivism. In particular, this would be an advantage for multivariate research designs with small samples and high numbers of variables. By matching groups on known explanatory variables, such as previous violence, drug abuse, and so forth, other variables were supposed to be given a possibility to emerge and inform our understanding of violence recidivism. However, our study did not identify possible explanations for the main finding of strong and significant differences in violent episodes between the Safe pilot group and the control group.

Due to the low number of forensic beds and low flow of patients in the mental health facilities we researched, recruitment took a long time, and we had to stop before we had reached a statistical power of about $80 \%$ that could guard against Type 1 or Type 2 errors. Obviously, we cannot criticize the matched pair design for this, and, for most other countries, this would not be a problem due to higher population rates in these forensic settings. Yet, the time and resources spent on recruitment must be balanced against the advantages of the matched pair design. In fact, we also question if the two comparison groups in a sense became too similar because of the matched design. Because we had almost no access to information about dynamic risk factors in the control group, our betweengroup comparison became limited. If feasible, an RCT 
study with a randomized, matched control design and repeated measurement of dynamic risk factors would be an ideal approach. Unfortunately, this was beyond what we could accomplish. Because of the low rates of forensic patients in Norway, it would be difficult to find a large enough pool to do a reliable randomization. In addition, there are methodological challenges and ethical obstacles against use of the RCT design in forensic psychiatry. Therefore, perhaps prospective naturalistic research with monitoring of risk management strategies and control of staff fidelity is a more viable approach.

\section{Strengths and limitations}

Thirty-six patients were involved in this investigation. This is a small sample, but taking into consideration that there were only around 200 forensic beds in Norway at the time of the study and that patient flow was low, the proportion of patients in the study was acceptable. Because of the small sample size, this research had high internal validity concerning information on historical risk factors, such as follow-up time, previous violence, and so forth. The same goes for the fact that the patients were matched for a series of these risk factors before the start of the study, and other possible risk factors were controlled for statistically after the deadline of data collection. In multiple regression the assumption of normally distributed errors is useful because when it holds true, one can make inferences about the regression parameters in the population that a sample was drawn from, even when the sample size is relatively small. Such inferences are usually made using significance tests and/or confidence intervals. While the significance level was acceptable $(p=0.020)$, the confidence interval was very wide. Therefore, even if the residuals had a normal distribution, one must be cautious in generalizing from this study to other studies and contexts (external validity). Including verbal and physical threats increased the incidence of violence from 9 to 61 episodes. There is always a likelihood of escalation from threats to physical assault, and this may provide important clinical information for risk management (e.g. ${ }^{23}$ ).

Naturally, there were also limitations. The most important sources of bias in non-randomized designs are selection bias and confounding. The inclusion of patients in the Safe pilot group followed the natural discharge process and procedure in the actual forensic unit. Still, as soon as they were assessed for a planned discharge within approximately one year, the Safe pilot project measurement started. For most of the patients, development and implementation of risk management strategies were also started. There were no extra treatment resources allocated. It is not very likely that the clinical staff who selected patients for inclusion in the Safe pilot project one year before discharge had any selection bias. The main idea of the project was to follow the natural patient flow. Even if the strict inclusion and matching criteria guarded against confounding variables, there is always the possibility that monitoring of violence and other variables may be confounded in naturalistic designs. Moreover, we did not measure the staff-patient interactions in the control group, and there is a likelihood that those in the treatment condition had more intensive clinical contact due to the repeated assessment of risk factors. Hence, we must be mindful that the intensity of the intervention may explain more than the specific content of it. Apart from a lower number of patients with personality disorders, the Safe pilot sample was similar to the Norwegian population of forensic mental health patients. The low statistical power of the study is perhaps the most disturbing limitation. Lack of information concerning implementation of risk management plans after discharge is another one, because we did not monitor the use of the written clinical information that followed the Safe pilot project patients when they were transferred to their new health service facility. Like with any naturalistic, prospective follow-up research, we did not have a complete overview of interpersonal changes in the patients' lives.

\section{Conclusion}

This matched pair naturalistic prospective research had two main aims. The first one was to compare the Safe pilot sample with a matched control group on violent recidivism after discharge from forensic mental health wards. Control group patients committed almost nine times more violent episodes and had a much higher rate of severe violence. Due to low statistical power and comprehensive matching of risk factors between the two groups, it was very difficult to find explanatory factors for this difference apart from that being in the Safe pilot group appeared to be protective against violence. Having a high number of risk management plans per patient was associated with violence during follow-up. This link was stronger for control patients. The second aim was to test whether the matched pair design was feasible and could help single out predictor variables of violence recidivism in a better way than, for instance, a case-control design would. Due to the time it took to recruit even a small sample of matched pairs and the low statistical power we obtained, we cannot recommend this design for use in 
small samples of forensic patients. The main lesson we have learned from this research is to do prospective monitoring, not only of occurred violence, but also of the implementation and use of risk management plans and other preventive measures.

\section{Acknowledgment}

We thank the clinical staff in the secure wards that were involved in this research project in the following institutions: Sandviken Psychiatric Hospital, Bergen Health Trust, Blakstad and Lier Psychiatric Hospitals, Vestre Viken Health Trust, and Gaustad Psychiatric Hospital, Oslo University Hospital Health Trust.

We are also grateful to John Olav Roaldset, $\mathrm{MD}, \mathrm{PhD}$ for converting antipsychotic medication dosages at discharge to chlorpromazine equivalents per day.

This research did not receive any specific grant from funding agencies in the public, commercial, or not-forprofit sectors

\section{Disclosure}

The authors report no conflicts of interest in this work.

\section{References}

1. Fazel S, Fiminska Z, Cocks C, Coid J. Patient outcomes following discharge from secure psychiatric hospitals: systematic review and meta-analysis. Br J Psychiatry. 2016;208(1):17-25. doi:10.1192/bjp. bp.114.149997

2. Coid J, Hickey N, Kahtan N, Zhang T, Yang M. Patients discharged from medium secure forensic psychiatry services: reconvictions and risk factors. $B r J$ Psychiatry. 2007;190:223-229. doi:10.1192/bjp. bp. 105.018788

3. Davies S, Clarke M, Hollin C, Duggan C. Long-term outcomes after discharge from medium secure care: a cause for concern. $\mathrm{Br} J$ Psychiatry. 2007;191:70-74. doi:10.1192/bjp.bp.106.029215

4. Hayes H, Kemp RI, Large MM, Nielssen OB. A 21-year retrospective outcome study of New South Wales forensic patients granted conditional and unconditional release. Aust N Z J Psychiatry. 2014;48 (3):259-282. doi:10.1177/0004867413507610

5. Bogaerts S, Spreen M, Ter Horst P, Gerlsma C. Predictive validity of the HKT-R risk assessment for two and five-year recidivism in a cohort of Dutch forensic psychiatric patients. Int J Offender Ther Comp Criminol. 2018;62(8):2259-2270. doi:10.1177/0306624X17717128

6. Jeandarme I, Habets P, Oei TI, Bogaerts S. Reconviction and revocation rates in flanders after medium security treatment. Int $J$ Law Psychiatry. 2016;47:45-52. doi:10.1016/j.ijlp.2016.02.033

7. Bertman-Pate LJ, Burnett DM, Thompson JW, Calhoun CJ Jr., Deland S, Fryou RM. The New Orleans forensic aftercare clinic: a seven year review of hospital discharged and jail diverted clients. Behav Sci Law. 2004;22(1):159-169. doi:10.1002/bsl.575
8. Parker GF. Outcomes of assertive community treatment in an NGRI conditional release program. J Am Acad Psychiatry Law. 2004;32 (3):291-303.

9. Vitacco MJ, Vauter R, Erickson SK, Ragatz L. Evaluating conditional release in not guilty by reason of insanity acquittees: a prospective follow-up study in Virginia. Law Hum Behav. 2014;38(4):346-356. doi:10.1037/lhb0000071

10. Doyle M, Power LA, Coid J, Kallis C, Ullrich S, Shaw J. Predicting post-discharge community violence in England and Wales using the HCR-20V3. Int $J$ Forensic Ment Health. 2014;13(2):140-147. doi:10.1080/14999013.2014.906517

11. Penney SR, Marshall LA, Simpson AI. The assessment of dynamic risk among forensic psychiatric patients transitioning to the community. Law Hum Behav. 2016;40(4):374-386. doi:10.1037/lhb0000183

12. Bjørkly S, Sandli CS, Moger TA, Stang J. A follow-up interview of patients eight years after discharge from a maximum security forensic psychiatry unit in Norway. Int J Forensic Ment Health. 2010;9 (4):343-353. doi:10.1080/14999013.2010.534698

13. Bjørkly S. Violent patients' warning signals and indications of coping failure as criteria for specific risk management strategies: an introduction to the SAFE project. Issues Forensic Psychol. 2004;5:114126.

14. Statistisk Sentralbyrå, SSB. 2015.

15. World Health Organization. The ICD-10 Classification of Mental and Behavioural Disorders: Clinical Descriptions and Diagnostic Guidelines. Geneva: World Health Organization; 1992.

16. Monahan J, Appelbaum PS. Reducing violence risk - diagnostically based clues from the macarthur violence risk assessment study. In: Hodgins S, editor. Violence among the Mentally iII - Effective Treatments and Management Strategies. Boston: Kluwer Academic Publishers; 2000:19-35.

17. Fluttert F, Van Meijel B, Webster C, Nijman H, Bartels A, Grypdonck M. Risk management by early recognition of warning signs in patients in forensic psychiatric care. Arch Psychiatr Nurs. 2008;22 (4):208-216. doi:10.1016/j.apnu.2007.06.012

18. Douglas KS, Hart SD, Webster CD, Belfrage H. HCR-20-V3 Assessing Risk for Violence - User Guide. Vancouver: Mental Health, Law, and Policy Institute, Simon Fraser University; 2013.

19. Charette Y, Crocker AG, Seto MC, Salem L, Nicholls TL, Caulet M. The national trajectory project of individuals found not criminally responsible on account of mental disorder in Canada. Part 4: criminal recidivism. Can J Psychiatry. 2015;60(3):127-134. doi:10.1177/ 070674371506000307

20. Penney SR, Marshall L, Simpson AI. A prospective study of pathways to hospital readmission in Canadian forensic psychiatric patients. $J$ Forens Psychiatry Psychol. 2018;29(3):368-386. doi:10.1080/14789949.2017.1395061

21. Jewell A, Cocks C, Cullen AE, Fahy T, Dean K. Predicting time to recall in patients conditionally released from a secure forensic hospital: a survival analysis. Eur Psychiatry. 2018;49:1-8. doi:10.1016/j. eurpsy.2017.11.005

22. Conlin A, Braham L. Comparison of outcomes of patients with personality disorder to patients with mental illness, following discharge from medium secure hospital: systematic review. $J$ Forens Psychiatry Psychol. 2018;29(1):124-145. doi:10.1080/ 14789949.2017.1347804

23. Mitchell M, Palk G, Kavanagh D. No idle threat: exploring threats and violence in persons with mental illness. Int J Forensic Ment Health. 2019;1-10. doi:10.1080/14999013.2018.1552636 


\section{Publish your work in this journal}

Psychology Research and Behavior Management is an international, peer-reviewed, open access journal focusing on the science of psychology and its application in behavior management to develop improved outcomes in the clinical, educational, sports and business arenas. Specific topics covered in the journal include: Neuroscience, memory and decision making; Behavior modification and management; Clinical applications; Business and sports performance management; Social and developmental studies; Animal studies. The manuscript management system is completely online and includes a very quick and fair peer-review system, which is all easy to use. Visit http://www. dovepress.com/testimonials.php to read real quotes from published authors.

Submit your manuscript here: https://www.dovepress.com/psychology-research-and-behavior-management-journal 